\title{
Prevalence and Incidence of HIV and Sexual Risk Behaviors in Crack Users in the San Salvador Metropolitan Area, El Salvador*
}

\author{
Julia Dickson-Gomez ${ }^{1}$, Julia Lechuga ${ }^{2}$, Laura Glasman ${ }^{1}$, Steven Pinkerton ${ }^{1}$, \\ Gloria Bodnar ${ }^{3}$, Pamela Klein ${ }^{1}$
}

${ }^{1}$ Center for AIDS Intervention Research, Department of Psychiatry and Behavioral Medicine, The Medical College of Wisconsin, Milwaukee, USA; ${ }^{2}$ University of Texas, El Paso, USA; ${ }^{3}$ Departamento de Investigación, Fundación Antidrogas de El Salvador (FUNDASALVA), San Salvador, El Salvador.

Email: jdickson@mcw.edu, julialec@utep.edu, lglasman@mcw.edu, pinkrton@mcw.edu, gbodnar@fundasalva.org, paklein@mow.edu

Received September $4^{\text {th }}, 2013$; revised October $4^{\text {th }}, 2013$; accepted October $11^{\text {th }}, 2013$

Copyright (C) 2013 Julia Dickson-Gomez et al. This is an open access article distributed under the Creative Commons Attribution License, which permits unrestricted use, distribution, and reproduction in any medium, provided the original work is properly cited.

\begin{abstract}
Objective: It is in order to estimate the prevalence and incidence of HIV, the frequency of sexual risk behaviors, and perceptions of available resources to prevent and treat HIV among crack users in the San Salvador Metropolitan Area. Methods: We conducted a survey of 420 crack users by using respondent-driven sampling to measure demographic characteristics, the quantity and frequency of drug use, history of STIs, including HIV, and experiences with organizations which provide prevention and treatment of HIV. Each participant offered a free and voluntary HIV test and was asked permission to share the results of the test with the study. Bernoullian modeling was used to estimate the prevalence and incidence of HIV among heterosexual males in this population. Results: The estimated prevalence was $7 \%$ (95\% CI: $2.3 \%-9.8 \%$ ) among participants who agreed to take the test and share the results, and $4.9 \%$ (95\% CI: $2.8 \%$ $7.8 \%$ ) assuming that those who did not take the test or share results were seronegative. Participants reported a high frequency of sexual risk behaviors. In addition, participants were reported to have little knowledge of organizations to prevent or treat HIV/AIDS; 58\% had never taken an HIV test prior to survey administration. Conclusions: Crack users in San Salvador are at high risk for HIV acquisition. HIV prevention interventions are urgently needed, especially interventions increasing access to HIV testing and prevention.
\end{abstract}

Keywords: Incidence and Prevalence of HIV; Sexual Risk Behavior; Crack; El Salvador

\section{Introduction}

Non-injection drug use has been associated with high prevalence of HIV and sexual risk behaviors such as sex exchanges for drugs or money, sex with multiple partners, sex without condoms and a high rate of sexual assault [1-4]. Crack, in particular, has been associated with high frequency of sex exchanges, lower prices for commercial sex work, diminished ability to negotiate condoms with clients and high rates of sexual assault $[2,3,5]$ due in part to the psychopharmacological effects of crack [5-9]. In spite of the strong research evidence linking crack use to HIV risk, crack use is not regularly monitored in surveil-

"Declaration of conflict of interest: the authors declare that they have conflicts of interest. lance of HIV and few HIV prevention interventions focus on crack users.

In El Salvador, data on the prevalence of crack use are sparse. In a national survey conducted in 2004, approximately $1 \%$ of the adult population had used crack in the past year, reaching a high of $4.9 \%$ among 18 years old people [10]. Among gang members, $65.7 \%$ were reported to use crack in the last month and $25.8 \%$ were reported to use crack daily [11]. A significant number of persons are affected directly by crack use through their own problematic use, or indirectly through the effects of drug sales and use within their communities [12-15].

El Salvador has the second-largest number of people reported to be living with HIV/AIDS in Central America [16], with an estimated overall prevalence of $0.8 \%$ [17]. 
However, the HIV epidemic is concentrated among vulnerable populations such as men who have sex with men (MSM; 10.8\% prevalence), female sex workers (FSW; $5.7 \%$ prevalence) and transgender women (19.7\% prevalence) [18]. Because the Ministry of Health does not collect data regarding non-injection drug use in national HIV surveillance data, the prevalence of HIV among crack users has not yet been estimated.

Through this study of crack users in the metropolitan area of San Salvador, El Salvador we aim to: 1) identify the frequency of sexual risk behaviors; and 2) explore crack users' knowledge of HIV and available HIV prevention and treatment programs; and 3) estimate the prevalence and incidence of HIV. The results of this study will aid in the development of interventions to prevent HIV transmission among non-injection drug users in El Salvador and other developing countries.

\section{Methods}

\subsection{Study Population and Recruitment}

Surveys were conducted with crack users who lived in the Metropolitan Area of San Salvador, El Salvador and were recruited using respondent driven sampling (RDS). RDS was chosen for the following reasons: 1) RDS has been shown to be effective in reaching hidden populations; and 2) With long enough chains of referrals from seeds, the composition of the sample becomes stable (reaches equilibrium), and reflective of the universe of crack users regardless of seed selection [19,20].

Field staff identified and recruited 3 initial "seeds". Field staff chose additional seeds when chains of referral were broken, resulting in a total of 22 total seeds who recruited 398 survey participants for a total sample size of 420 participants. Eligibility criteria included residing in, or using or buying drugs in one of the 7 low-income communities selected for study, being 16 years old or older, and having smoked crack in the last 2 weeks. After completing the survey, seeds were given 3 coupons to recruit other members of their social network. Eligible recruits who completed the survey were also given 3 coupons to recruit members of their social networks resulting in several waves of recruitment. Dual incentives were given to encourage recruitment, $\$ 5$ for participating in the survey, and \$2 for each participant successfully recruited into the project.

All participants were read and signed an informed consent forms in Spanish. The research was approved by Institutional Review Boards at the Institute for Community Research, Hartford CT, the Medical College of Wisconsin, Milwaukee, WI, and the Universidad Centroamericana Jose Simeon Cañas, San Salvador, El Salvador.

\subsection{Survey Measures}

Surveys were conducted face to face by trained interviewers and were approximately 90 minutes in duration. Demographic factors measured included age, gender, marital status, monthly income, type of employment (formal or informal), and level of education. Participants self-reported if they worked in the formal or informal economies. Formal work refers to work that is legally recognized and from which social security is collected and other income in paid. Informal work includes both illegal activities (drug selling, commercial sex work) and work that would otherwise be legal but which is unregulated by any government institution [21,22].

The survey included questions about lifetime substance use, including alcohol, crack, marijuana, sniffed cocaine, injected cocaine, sniffed heroin, injected heroin, nevados (marihuana with cocaine), bañados (marihuana with crack), and ecstasy. For each substance for which participant reported use, the participants were asked the age at which they first started using the substance, and the frequency and quantity of substance use (number of days in the last month, number of times in the last month, and number of times in the day prior to survey administration).

Sexual risk behaviors were measured for the 30 days prior to survey administration and included the number of: sexual partners for anal, oral and vaginal sex acts; sex acts in exchange for money; sex acts in exchange for crack; sex acts in a location where crack is used; sex acts with a drug user; and sex acts under the effect of alcohol or drugs. For each risk behavior, participants were asked to provide the overall number of sexual partners/acts and the number of sexual partners/acts without a condom. Self-reported STI diagnosis history, including the specific STI diagnosed from a list of common STIs, was also collected. HIV testing history was also ascertained, including whether the participant ever tested for HIV, the last time the participant took an HIV test in months and years, and whether they ever received an HIV/AIDS diagnosis from a doctor.

Survey participants were asked if they were aware of organizations that prevented or treated HIV/AIDS or drug dependence, and whether they had ever received information, talks or interventions about the prevention or treatment of HIV or drug dependence. Participants were asked if they knew of places where they could obtain condoms, and to indicate the types of places they received a condom from a list.

After the survey, all participants were offered a free, voluntary HIV test. Participants could choose not to take an HIV test, to take the test and not share the results with the project, or to take the test and share results with the project. Staff from FUNDASIDA, a non-governmental 
AIDS Service Organization, administered the test, pre and post-test counseling. Participants' decisions regarding whether to take the test and share results were recorded, as were test results of participants' who gave their consent to share results with the project.

\subsection{Statistical Analyses}

Demographic variables, risk behaviors, substance use, and knowledge of available services for the prevention and treatment of HIV/AIDS or substance use were evaluated using frequency tables, and means and standard deviations where appropriate.

HIV prevalence was calculated among all survey participants using the Respondent Driven Sampling Analysis Tool 5.6 [23], developed specifically for analyzing samples recruited through RDS. This analysis program estimates the prevalence of HIV within a specific population (i.e. crack users) based upon percent of persons living with HIV in the sample, after adjustment for: 1) the number of crack users with whom the participant regularly interacted; and 2) the HIV status of the person who recruited the participant. The RDS network data and proportional recruitment strategy across groups can then be used to make unbiased population estimates [19].

HIV incidence rates were estimated among a subsample of men who reported having heterosexual vaginal or anal intercourse in the previous month $(\mathrm{n}=237)$. Men who reported male-male intercourse in addition to heterosexual intercourse were excluded from the analyses (n $=141,43 \%$ of all men). Men with male-male intercourse were excluded because the survey did not collect information on whether participants engaged in receptive or insertive anal intercourse and these have much different risks for acquiring HIV.

A Bernoullian model of HIV transmission [24] was used to estimate each male participants' monthly risk of acquiring HIV through vaginal and anal intercourse, based on the sexual behavior data collected in the main trial:

$$
\text { Monthly risk }=1-\left\{1-\{(1-\pi)\}+\pi\left(1-\alpha_{k}\right)^{k}\left(1-\alpha_{n}\right)^{n}\right\}^{m}
$$

where $m$ is the number of female partners reported by the participant; $\alpha_{k}$ and $\alpha_{n}=(1-\varepsilon) \alpha_{k}$ are the per-act female-to-male transmission probabilities for unprotected and condom-protected intercourse, respectively; $\varepsilon$ denotes the effectiveness of condoms at reducing HIV transmission; and $\pi$ is the prevalence of HIV infection among the female partners of study participants. This monthly risk corresponds to an annual risk of

Annual risk $=1-(1-\text { Monthly risk })^{12}$.

The expected HIV incidence among men in the study was estimated by calculating the mean annual risk across study participants.

Values for the number of partners $(m)$ and number of sex acts with and without condoms $(i, j, k$, and $n)$ were drawn from the main study survey. The following values were assumed for the remaining parameters per-act female-to-male transmission probability for unprotected intercourse, $\alpha_{k}=0.0038$; and condom effectiveness, $\varepsilon$ $=0.85$ [24]. Values for HIV prevalence were obtained from the estimates calculated as described above. The average incidence rate for men in the study sample was calculated together with lower and upper bound estimates.

\section{Results}

Ninety-two percent of the 420 survey participants were men $(n=386 / 420)$. The median age of the participants was 37.8 years (standard deviation: 9.4; range $18-63$ years). Most participants reported low levels of educational attainment; $41 \%$ attended primary school, $29.5 \%$ attended some secondary school, and only $21.9 \%$ finished high school. While $83 \%$ reported being currently employed, only $26 \%$ were employed in the formal sector (Table 1).

High levels of alcohol and marihuana use were reported by the survey participants; $99 \%$ of participants

Table 1. Demographics.

\begin{tabular}{lcc}
\hline & $\mathrm{N}$ & $\%$ \\
\hline Age (mean, std dev) & 37 & 9.6 \\
Gender & 386 & 92 \\
Male & 34 & 8 \\
Female & & \\
Marital Status & 67 & 16 \\
Married & 164 & 39 \\
Divorced & 180 & 43 \\
Single & 252 & 200 \\
Monthly Income (mean, median) & & \\
Type of Employment & 109 & 30 \\
Formal & 311 & 70 \\
Informal & & \\
Education & 173 & 41 \\
Primary School & 92 & 30 \\
Secondary School & & 18 \\
High School & & \\
Post-High School & & \\
\hline
\end{tabular}


reported ever using alcohol and $96.4 \%$ reported ever using marihuana (Table 2). After crack, alcohol was the most frequently used substance (median: 17 days in prior month). Few participants reported sniffing or injecting heroin or cocaine and none had reported doing so in the last 30 days.

Participants reported a high frequency of sexual risk behaviors in the 30 days prior to survey administration. On average, survey participants experienced 7 sex acts without condoms in the last 30 days (Table 3). In the month prior to survey administration, $28.3 \%$ of participants had sex with 3 or more partners, $13.5 \%$ exchanged sex for crack, $18.5 \%$ exchanged sex for money, and $42.4 \%$ had sex while under the influence of drugs or alcohol.

Nearly half of participants $(49.5 \%, n=208 / 420)$ reported receiving an STI diagnosis during their lifetimes. Of those who with a history of STIs, $53.8 \%$ were infected with an STI on more than one occasion $(n=112$ / 208). The most common STI reported by participants was gonorrhea $(30.5 \%, \mathrm{n}=63 / 208)$.

Only $15 \%$ participants reported knowledge of organizations that offered HIV prevention programs $(n=63$ / 420 ), and $10 \%$ knew of organizations to treat HIV ( $\mathrm{n}=$ 42/420). Of those with knowledge of such organizations, $68.2 \%$ reported receipt of information, talks or workshops to prevent or treat HIV $(n=43 / 63)$. The types of organizations that offered services were identified as government organizations (51.1\%), non-governmental organizations (33.3\%) and religious organizations (11.1\%). Over half of participants (66.4\%) reported obtaining condoms in the last 6 months; pharmacies (where condoms must be bought) and Ministry of Health clinics (where condoms are free) were the most frequently mentioned locations of condom acquisition (Table 4).

Only $42 \%$ of participants reported every having had an
HIV test. The most frequently mentioned locations where HIV tests were received were Ministry of Health clinics (58.5\%). Among participants who had been tested for HIV, the mean time since their most recent HIV test was 5 months.

Of the 22 RDS seeds, 16 tested negative for HIV and 6 elected not to take the test or share their results. Among the remaining, non-seed participants, $20(5 \%)$ either selfreported or tested HIV-positive, 236 (59.1\%) tested negative for HIV, and $142(35.6 \%)$ elected not to take the test or share their results. Over $50 \%(54.5 \%, \mathrm{n}=5 / 11)$ of participants who self-reported as HIV-positive also reported receipt of HIV treatment. All participants who reported or were tested HIV-positive were recruited to our study by participants who tested HIV-negative. Among those with a known HIV-test result, the estimated HIV prevalence was $7 \%(90 \%$ confidence interval [CI]: $2.3 \%$ $9.8 \%$ ). If we assumed that those were not tested for HIV or chose not to share their results were HIV negative, the estimated prevalence of HIV was $4.9 \%$ (90\% CI: $2.8 \%$ $7.8 \%)$.

HIV incidence rates were estimated for a subsample of heterosexual male survey participants. The average incidence rate was 0.0022 HIV infections per month (standard deviation $=0.0034$ ), with a range of 0.000 to 0.0300 HIV infections per month for individual study participants (Figure 1). The average annual incidence rate was $2.7 \%$. Sensitivity analyses varying the HIV prevalence of the female partners of the male participants yielded average incidence rates of $1.6 \%(\pi=2.8 \%)$ to $5.2 \%(\pi=$ $9.8 \%)$.

\section{Discussion}

Globally, the use of non-injection drugs, especially crack, has been associated with high rates of HIV and high risk

Table 2. Quantity and frequency of drug and alcohol use among crack users in San Salvador, El Salvador.

\begin{tabular}{|c|c|c|c|c|c|c|c|c|c|c|}
\hline & & & \multicolumn{2}{|c|}{ Age First Used } & \multicolumn{2}{|c|}{ Days in Last Month } & \multicolumn{2}{|c|}{ Times in Last Month } & \multicolumn{2}{|c|}{ Times Used Yesterday } \\
\hline & $\mathrm{N}$ & $\%$ & Median & $\mathrm{SD}$ & Median & $\mathrm{SD}$ & Median & $\mathrm{SD}$ & Median & $\mathrm{SD}$ \\
\hline Crack & 414 & 98.6 & 21 & 9.06 & 30 & 10.98 & 240 & 424.8 & 5 & 15.62 \\
\hline Alcohol & 416 & 99.0 & 14 & 4.75 & 17 & 12.06 & 240 & 1323.40 & 4 & 36.01 \\
\hline Marihuana & 405 & 96.4 & 15 & 6.54 & 3 & 12.50 & 6 & 109.68 & 0 & 2.93 \\
\hline Bañados & 348 & 82.9 & 21 & 9.91 & 4 & 11.72 & 6 & 110.02 & 0 & 3.47 \\
\hline Sniffed Cocaine & 329 & 21.7 & 20 & 7.30 & 0 & 6.12 & 0 & 19.08 & 0 & 0.62 \\
\hline Injected Cocaine & 39 & 9.3 & 20 & 8.40 & 0 & 0.34 & 0 & 0.35 & 0 & 0 \\
\hline Sniffed Heroine & 32 & 7.6 & 22 & 6.47 & 0 & 3.77 & 0 & 18.85 & 0 & 0.89 \\
\hline Injected Heroine & 35 & 8.3 & 19 & 6.75 & 0 & 0 & 0 & 0 & 0 & 0 \\
\hline
\end{tabular}

Note. $\mathrm{N}=$ number of participants ever used. $\%=$ percentage of participants ever used. 
Table 3. Frequency of sexual risk behaviors among crack users in San Salvador, El Salvador in $\mathbf{3 0}$ days prior to survey administration.

\begin{tabular}{ccccc}
\hline \multicolumn{2}{c}{ Overall } & \multicolumn{2}{c}{ Without a Condom } \\
\hline $\mathrm{N}$ & $\%$ & $\mathrm{~N}$ & $\%$
\end{tabular}

Number of unprotected sex acts

$\begin{array}{ccccc}0 \text { acts } & - & - & 172 & 40.9 \\ 1-2 \text { acts } & - & - & 35 & 8.4 \\ \geq 3 \text { acts } & - & - & 209 & 50.2\end{array}$

Number of sex partners

$\begin{array}{ccccc}0 \text { partners } & 120 & 28.6 & 182 & 43.3 \\ 1-2 \text { partners } & 182 & 43.3 & 172 & 41 \\ \geq 3 \text { partners } & 118 & 28.1 & 66 & 15.7\end{array}$

Sex in exchange for crack

$\begin{array}{ccccc}0 \text { acts } & 351 & 83.6 & 365 & 86.9 \\ 1-2 \text { acts } & 12 & 2.9 & 7 & 1.7 \\ \geq 3 \text { acts } & 57 & 13.6 & 48 & 11.4\end{array}$

Sex in exchange for money

$\begin{array}{ccccc}0 \text { acts } & 320 & 76.2 & 356 & 84.8 \\ 1-2 \text { acts } & 23 & 5.5 & 18 & 4.3 \\ \geq 3 \text { acts } & 77 & 18.3 & 46 & 11.0\end{array}$

Sex while drunk or high

\begin{tabular}{ccccc}
0 acts & 208 & 49.5 & 252 & 60 \\
$1-2$ acts & 35 & 8.3 & 33 & 7.9 \\
$\geq 3$ acts & 177 & 42.1 & 135 & 32.1 \\
\hline
\end{tabular}

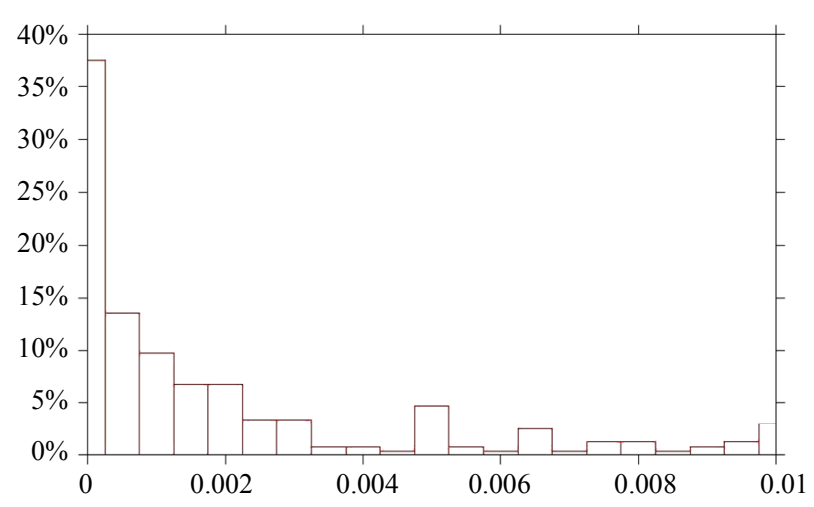

Figure 1. Distribution of monthly risk of HIV infection among crack users in the San Salvador metropolitan area, El Salvador.

sexual behaviors. Despite having one of the largest HIV epidemics in Central America, the prevalence of HIV and
Table 4. Places where crack users reported obtaining condoms.

\begin{tabular}{lcc}
\hline & $\mathrm{N}$ & $\%$ \\
\hline Places & 87 & 68.6 \\
Pharmacy & 6 & 2.2 \\
Supermarket & 11 & 4.0 \\
Gas Station & 9 & 3.2 \\
Store & 155 & 56.0 \\
Government Health Clinic & 14 & 5.1 \\
Private Clinic & 9 & 3.2 \\
Hospital & 9 & 2.9 \\
Mobile Van & 43 & 15.5 \\
NGO & 14 & 5.1 \\
Bar/Brothel & & \\
People & 18 & 6.5 \\
Community Outreach Worker & 58 & 13.8 \\
Friends & 26 & 9.4 \\
Artner & 2 & 1.0 \\
Family & 518 \\
\hline
\end{tabular}

Note. Percentages based only on participants who reported obtaining condoms in the last 6 months $(\mathrm{N}=279)$.

high risk sexual behaviors among crack users has not yet been investigated. To address this gap, we conducted a survey among crack users in the San Salvador metropolitan region of El Salvador to assess the frequency of high risk sexual behaviors, knowledge of and access to HIV and drug prevention services, and estimate the HIV prevalence and incidence in this population. Crack users in San Salvador are at high risk of HIV acquisition due to sexual behaviors such as sex for money or crack exchanges, sex with multiple partners, and sex without condoms, as well as the high prevalence of HIV in the population.

The estimated HIV prevalence among crack users in San Salvador was $4.9 \%-7.0 \%$, which was similar to the prevalence of HIV among sex workers [18]. In addition to prevention efforts targeted towards commercial sex workers, transgender women and men who have sex with men, prevention interventions must also be developed and targeted specifically for non-injection drug users. While many male and female commercial sex workers also use crack, existing prevention efforts will not work for all crack users, particularly those who do not identify as sex workers because they pay for, rather than are paid for, sex exchanges, or because they exchange sex on a 
sporadic basis $[25,26]$. Based on mathematical modeling and our respondent driven sample design, the average annual HIV incidence among heterosexual males was estimated to be $2.7 \%$, which suggests that failing to intervene with this population could cause an alarming increase in the incidence of HIV/AIDS. In scenarios with higher rates of HIV prevalence in female partners of heterosexual males, the annual average incidence could be as high as $5.2 \%$.

Knowledge of and access to HIV prevention and treatment resources among non-injection drug users in El Salvador was low. Over half of participants had never before been tested for HIV. Very few participants were aware of organizations that worked to prevention HIV $(15 \%)$ or offered HIV treatment $(10 \%)$ in their communities. This low level of awareness of HIV prevention and treatment resources may explain the low prevalence of HIV testing among this population.

Future research should examine whether the lack of knowledge regarding specific organizations that treat HIV/AIDS in the community is related to a general lack of knowledge of the existence of free and effective HIV treatment. In-depth interviews with substance-using people living with HIV in El Salvador revealed that many persons had no knowledge of antiretroviral therapy before their diagnosis, received little information about the importance of linking into medical care, and still believed that HIV was a death sentence (publication forthcoming). While improved HIV counseling and testing may increase the proportion of substance using persons living with HIV who link to medical treatment, many non-injection drug users may still be reluctant to test for HIV unless general knowledge of effective treatments is increased.

Access to condoms in the San Salvador Metropolitan Area was inadequate; only $66.4 \%$ of participants reported that they had bought or obtained condoms. Most often, participants reported buying condoms from businesses. However, a high percentage also reported obtaining free condoms from government health clinics. Importantly, few participants received condoms through community outreach workers. Community outreach might reduce barriers to obtaining condoms related to stigma (condoms must be requested from Ministry of Health clinics) and affordability [26]. In turn, improved access may increase condom use in this population.

Crack users reported high rates of risky sexual behaviors. In the month prior to survey administration, over half of the participants reported having had sex without a condom on more than 3 occasions; $28.3 \%$ had more than one sexual partner, and $16.4 \%$ exchanged sex for crack or money. Over half reported having been diagnosed with an STI in the last year, putting them at even greater risk for becoming infected with HIV [27-29]. Efforts to increase timely STI screening and treatment in places that are accessible and acceptable to crack users, e.g. clinics, soup kitchens or shelters, may reduce the risk of HIV transmission.

The majority of survey variables - except for the results of HIV tests-was self-reported and could have been influenced by social desirability bias. However, a survey that resulted in self-reported measures was the most viable way to access sexual and drug use behavior data on this traditionally hidden and stigmatized population. In spite of our efforts to recruit female crack users, nearly all survey participants were male, which limits the generalizability of our results. Further, due to the small numbers of female crack users, HIV incidence could only be estimated for males.

\section{Conclusion}

Crack users are at especially high risk for HIV acquisition. However, national surveillance in El Salvador neglects to monitor this key and vulnerable population. Our study addresses this important knowledge gap, estimating HIV prevalence and incidence among crack users in San Salvador. Given their high rates of risky sexual behavior and drug use, as well as lack of knowledge of available HIV prevention and treatment options, crack users in San Salvador are especially vulnerable to acquisition of HIV and other STIs. Improved surveillance and prevention efforts tailored to the needs of this high risk population are needed to mitigate the HIV and drug use epidemics in San Salvador.

\section{Acknowledgements}

We wish to thank the FUNDASALVA research team, without whom this study could not have been possible, and the support of the Universidad Jose Simeón Cañas de El Salvador. This Project was funded by the National Institutes of Health (R01 DA020350 and P30 MH57226).

\section{REFERENCES}

[1] S. Strathdee and J. K. Stockman, "Epidemiology of HIV among Injecting and Non-Injecting Drug Users: Current Trends and Implications for Interventions," Current HIV/ AIDS Reports, Vol. 7, No. 2, 2010, pp. 99-106. http://dx.doi.org/10.1007/s11904-010-0043-7

[2] J. A. Inciardi, "Crack, Crack House Sex, and HIV Risk," Archives of Sexual Behavior, Vol. 24, No. 3, 1995, pp. 249-269.

http://dx.doi.org/10.1007/BF01541599

[3] M. Ratner, "Sex, Drugs, and Public Policy: Studying and Understanding the Sex-For-Crack Phenomenon," In: M. Ratner, Ed., Crack Pipe as Pimp: An Ethnographic Investigation of Sex-for-Crack Exchanges, Lexington Books, 
New York, 1993.

[4] R. Falck, et al., "The Epidemiology of Physical Attack and Rape among Crack-Using Women," Violence and Victims, Vol. 16, No. 1, 2001, pp. 79-89.

[5] D. Jones, et al., "The High-Risk Sexual Practices of CrackSmoking Sex Workers Recruited from the Streets of Three American Cities," Sexually Transmitted Diseases, Vol. 25, No. 4, 1998, pp. 187-193. http://dx.doi.org/10.1097/00007435-199804000-00002

[6] J. A. Inciardi, "Kingrats, Chicken Heads, Slow Necks, Freaks, and Blood Suckers: A Glimpse at the Miami SexFor-Crack Market," In: M. Ratner, Ed., Crack Pipe as Pimp: An Ethnographic Investigation of Sex-for-Crack Exchanges, Lexington Books, New York, 1993.

[7] J. A. Inciardi, "Trading Sex for Crack among Juvenile Crack Users: A Research Note," Contemporary Drug Problems, Vol. 16, No. 4, 1989, pp. 689-700.

[8] L. Maher, "Sexed Work: Gender, Race, and Resistance in a Brooklyn Drug Market," Clarendon Press, Oxford, 1997.

[9] P. Bourgois and E. Dunlap, "Exorcising Sex-For-Crack: An Ethnographic Perspective from Harlem," In: M. Ratner, Ed., Crack Pipe as Pimp: An Ethnographic Investigation of Sex-fro-Crack Exchanges, Lexington Books, New York, 1993.

[10] Organization of American States, "Informe Preliminario PACARDO El Salvador," 2002.

[11] M. Santacruz Giralt and A. Concha-Eastman, "Barrio Adentro: La Solidaridad Violenta de las Pandillas," OAS, Istituto Universitario de Opinión Pública, San Salvador, 2001.

[12] J. Dickson-Gomez, "Structural Factors Influencing Patterns of Drug Selling and Use in the San Salvador Metropolitan Area," Medical Anthropology Quarterly, Vol. 24, No. 2, 2010, pp. 157-181.

http://dx.doi.org/10.1111/j.1548-1387.2010.01095.x

[13] J. Dickson-Gomez, "Substance Abuse Disorders Treatment in El Salvador: Analysis of Policy Making Related Failure," Substance Use and Misuse, in Press.

[14] J. Dickson-Gomez, et al., "With God's Help I Can Do It: Crack Users' Formal and Informal Recovery Experiences in El Salvador," Substance Use and Misuse, Vol. 46, No. 4, 2010, pp. 426-439. http://dx.doi.org/10.3109/10826084.2010.495762

[15] J. Dickson-Gomez, et al., "The Relationship between Community Structural Characteristics, the Context of Drug Use, and HIV Risk Behaviors in San Salvador, El Salvador," Substance Use and Misuse, Vol. 47, No. 3, 2012, pp. 265-277. http://dx.doi.org/10.3109/10826084.2011.635325

[16] UNAIDS, "Global Report: UNAIDS Report on the Global Epidemic,” 2010.

[17] CARE Working Group and Department for International Health/SNHS, “Central American Regional Report,” 2010.
[18] Ministerio de Salud Publica y Asistencia Social, "Plan Estratégico Nacional Multisectorial de la Respeusta al VIH/SIDA e ITS 2011-2015," CONASIDA, MSPAS, San Salvador, 2011.

[19] D. D. Heckathorn and R. Magnani, "Snowball and Respondent-Driven Sampling," In: Behavioral Surveillance Surveys: Guidelines for Repeated Behavioral Surveys in Populations at Risk of HIV, in Press.

[20] D. D. Heckathorn, et al., "Extensions of Respondent Driven Sampling: A New Approach to the Study of Injection Drug Users Aged 18 - 25," AIDS and Behavior, Vol. 6, No. 1, 2002, pp. 55-67. http://dx.doi.org/10.1023/A:1014528612685

[21] M. Castells and A. Portes, "World Underneath: The Origins, Dynamics, and Effects of the Informal Economy," In: A. Portes, M. Castells and L. Benton, Eds., The Informal Economy: Studies in Advanced and Less Developed Countries, Johns Hopkins University Press, Baltimore, 1989.

[22] J. Dickson-Gomez, et al., "Hustling and Housing: Drug Users' Strategies to Obtain Shelter and Income in Hartford, CT," Human Organization, Vol. 68, No. 3, 2009, pp. 269-279.

[23] D. D. Heckathorn and E. Volz, "Respondent-Driven Sampling Analysis Tool (RDSAT) 5.6.0," Department of Sociology, Cornell University, Ithaca, 2006.

[24] S. D. Pinkerton and P. R. Abramson, "Effectiveness of Condoms in Preventing HIV Transmission," Social Science and Medicine, Vol. 44, No. 19, 1997, pp. 13031312. http://dx.doi.org/10.1016/S0277-9536(96)00258-4

[25] J. Dickson-Gomez, et al., "Crack Use Sites and HIV in El Salvador," Journal of Drug Issues, Vol. 37, No. 2, 2007, pp. 445-476. http://dx.doi.org/10.1177/002204260703700211

[26] J. Dickson-Gomez, et al., "Resources and Obstacles to Developing and Implementing a Structural Intervention to Prevent HIV in San Salvador, El Salvador," Social Science and Medicine, Vol. 70, No. 3, 2010, pp. 351-359. http://dx.doi.org/10.1016/j.socscimed.2009.10.029

[27] B. Van Der Pol, et al., "Trichomonosas Vaginalis Infection and Human Immunodeficiency Virus Acquisition in African Women," Journal of Infectious Diseases, Vol. 197, No. 4, 2008, pp. 548-554. http://dx.doi.org/10.1086/526496

[28] H. Burris, et al., "Getting Research into Policy-Herpes Simplex Virus Type-2 (HSV-2) Treatment and HIV Infection: International Guidelines Formulation and the Case of Ghana," Health Research Policy Systems, Vol. 9, Suppl. 1, 2011, p. S5. http://dx.doi.org/10.1186/1478-4505-9-S1-S5

[29] N. Chkarishivili, et al., "High Seroprevalence of Chlamydia Trachomatis in Newly Diagnosed Human Immunodeficienty Virus Patients in Georgia," Georgian Medical News, Vol. 189, 2010, pp. 12-16. 